\title{
Enfermedad de Chagas en México
}

\author{
Julieta Rojo-Medina, ${ }^{1}$ Cuitláhuac Ruiz-Matus, ${ }^{2}$ Paz María Salazar-Schettino \\ y Jesús Felipe González-Roldán ${ }^{4}$ \\ 'Secretaría de Salud, Centro Nacional de la Transfusión Sanguínea, Dirección General, Ciudad de México, México; ${ }^{2}$ Secretaría de Salud, Dirección \\ General de Epidemiologia, Ciudad de México, México; ${ }^{3}$ Universidad Nacional Autónoma de México, Facultad de Medicina, Departamento de \\ Microbiología y Parasitología; Ciudad de México, México; ${ }^{4}$ Secretaría de Salud, Centro Nacional de Programas Preventivos y Control de \\ Enfermedades, Dirección General, Ciudad de México, México
}

\section{Resumen}

La enfermedad de Chagas, causada por el Trypanosoma cruzi, está considerada como la parasitosis más grave en América. Se transmite principalmente por triatominos (chinches). El doctor Mazzoti reportó los dos primeros casos humanos en México. La forma de transmisión es por la entrada al organismo de los parásitos en heces del insecto, por transfusión sanguínea, de madre a hijo, por trasplante de órganos y por accidentes de laboratorio. En México se estima que 1.1 millones de personas están infectadas; la incidencia en 2012 fue de 0.70 por 100000 habitantes. En 2017, las mayores tasas de incidencia se registraron en Yucatán, Oaxaca e Hidalgo. La infección ocasiona miocardiopatías y megaórganos del tracto digestivo. El diagnóstico en fase aguda es por abordaje parasitológico y en fase crónica, por estudios de tamizaje por laboratorio. En los bancos de sangre de México, el estudio de la enfermedad de Chagas es de observancia obligatoria; de 2007 a 2016, la seroprevalencia ha disminuido de 0.40 a 0.32 debido a la mejora de los procesos de selección al donante y al cuestionario ad hoc. Los blancos del parásito son las células neuronales y las de los músculos liso y miocárdico. La asociación de la destrucción neuronal y del músculo liso define la presentación de los síndromes megachagásicos. Las manifestaciones iniciales de la enfermedad pueden pasar desapercibidas; $5 \%$ de los pacientes presenta signos y síntomas aparentes y $30 \%$ evolucionará a la fase crónica asintomática. Los tratamientos actuales tienen efecto en la fase aguda. Para el control de la enfermedad de Chagas se dispone del Programa de Acción Específico para la Vigilancia Prevención y Control de la Enfermedad de Chagas (PAE Chagas 2013-2018), encaminado a eliminar la transmisión transfusional y congénita y a controlar la transmisión vectorial. De la detección oportuna, el tratamiento etiológico temprano y la ampliación de cobertura depende el éxito de la atención médica. Por su parte, la vigilancia y tamizaje de las mujeres embarazadas que viven en zonas de riesgo y el tamizaje universal de donadores de sangre y órganos harán posible la eliminación de la transmisión connatal y transfusional.

PALABRAS CLAVE: Enfermedad de Chagas. Trypanosoma cruzi. Triatominos.

\section{Abstract}

Chagas disease, which is caused by Trypanosoma cruzi, is considered to be the most serious parasitic disease in America. It is transmitted mainly by triatominae ("kissing bugs"). Mazzoti reported the first two human cases in Mexico. The form of transmission is by parasites entering the organism in feces of the insect, by blood transfusion, from mother to child, by organ transplant and laboratory accidents. In Mexico, 1.1 million people are estimated to be infected; the incidence in 2012 was 0.70 per 1,00,000 population. In 2017, the highest incidence rates were registered in Yucatán, Oaxaca and Hidalgo. The infection causes cardiomyopathies and mega-organs of the digestive tract. Diagnosis in the acute phase is by parasitological approach and, in the chronic phase, by laboratory screening studies. In Mexico's blood banks, screening for Chagas disease is mandatory; from 2007 to 2016, seroprevalence has decreased from 0.40 to 0.32 due to the improvement of donor selection processes and the ad hoc questionnaire. The targets of the parasite are neurons and smooth and myocardial muscle cells. The

Fecha de recepción: 24-07-2018

Fecha de aceptación: 25-07-2018

DOI: 10.24875/GMM.18004515
Gac Med Mex. 2018;154:605-612

Disponible en PubMed

www.gacetamedicademexico.com 
association of neuronal and smooth muscle destruction defines the presentation of chagas mega-syndromes. Initial manifestations of the disease can go unnoticed; $5 \%$ show apparent signs and symptoms and $30 \%$ will progress to the chronic asymptomatic phase. Currently available treatments have effect in the acute phase. For the control of Chagas disease, the Specific Action Program for the Prevention and Control of Chagas Disease (PAE Chagas 2013-2018) is available to initiate activities aimed at eliminating transfusion and congenital transmission and controlling vector transmission. The success of medical care depends on oportune detection, early etiological treatment and coverage broadening. On the other hand, monitoring and screening of pregnant women living in risk areas and blood and organ donors universal screening will enable the elimination congenital and transfusion transmission.

KEY WORDS: Chagas disease. Trypanosoma cruzi. Triatominae.

\section{Panorama epidemiológico de la enfermedad de Chagas en México}

La tripanosomiasis americana o enfermedad de Chagas está considerada la parasitosis más grave en América y es una de las 14 enfermedades del rezago en la lista de la Organización Mundial de la Salud. ${ }^{1}$

En 1909, Carlos Ribeiro Justiniano Chagas identificó por primera vez al parásito, describió los insectos transmisores y detalló algunos síntomas, incluyendo alteraciones cardiacas y del sistema nervioso. Originalmente, Carlos Chagas nombró al parásito causante de esta enfermedad como Schizotrypanum cruzi. ${ }^{2}$

En 1928, Hoffman logró correlacionar por primera vez al triatomino como transmisor de Chagas y en 1940, el doctor Luis Mazzoti reportó los dos primeros casos humanos en México. ${ }^{3}$

La tripanosomiasis americana o enfermedad de Chagas es causada por el parásito protozoo Trypanosoma cruzi. La infección se transmite principalmente por triatominos de la familia Reduviidae, orden Hemiptera (chinches), subfamilia Triatominae. Se transmite principalmente por contacto con las heces u orina infectadas de insectos triatomíneos que se alimentan de sangre. Estos insectos viven en las grietas y huecos de las paredes y los tejados de las casas en las zonas rurales y suburbanas. Permanecen ocultos durante el día, por la noche entran en actividad, pican en una zona expuesta de la piel y defecan cerca de la picadura. Los parásitos penetran en el organismo cuando la persona se rasca y empuja las heces o la orina hacia la picadura, los ojos, la boca o alguna lesión cutánea abierta. Existen otras formas de transmisión: por consumo de alimentos contaminados por T. cruzi, transfusión de sangre, de madre infectada a hijo, trasplante de órganos y por accidentes de laboratorio. ${ }^{4}$

Este padecimiento se encuentra distribuido principalmente en América Latina, pero en las últimas décadas se ha observado en Estados Unidos, Canadá, en 17 países europeos y algunos del Pacífico occidental. En México se logró la eliminación de Rhodnius prolixus como principal vector entre 2009 y 2010 . El $20 \%$ de la población latinoamericana se considera en riesgo de adquirir la infección, sobre todo en las zonas endémicas de los países de la región. ${ }^{5}$

En México se considera un problema de salud pública ya que se estima 1.1 millones de personas infectadas. ${ }^{6}$ La incidencia de 2000 a 2007 se mantuvo en un rango de 0.07 a 0.37 por 100 mil habitantes; a partir de 2008 ascendió de 0.61 a 0.70 por 100 mil habitantes en 2012.

En 2015, a través del Sistema Nacional de la Secretaría de Salud, en México se detectaron ocho géneros y 31 especies de triatoma. En dos terceras partes del territorio mexicano existen las condiciones para que se lleve a cabo la transmisión vectorial.

Las especies intradomiciliarias son Triatoma barberi, asociada con miocardiopatías y megas (dilataciones) de órganos del tracto digestivo, y Triatoma dimidiata, distribuida desde los países andinos y de Centroamérica hasta México, asociada con cardiopatías. Las principales especies identificadas en 2015 en la República Mexicana fueron Triatoma longipenmis (34\%), Triatoma pallidipennis $(23 \%)$ y Triatoma dimidiata (29\%) (Figura 1$)$.

La Dirección General de Epidemiología de la Secretaría de Salud realiza, a través del Sistema Nacional de Vigilancia Epidemiológica, el monitoreo de la tripanosomiasis aguda y crónica, que debe notificarse de forma inmediata.

La notificación de casos presenta una tendencia ascendente hasta 2015 , debido al registro de 53 casos retroactivos provenientes del Estado de México. Durante 2018, hasta abril, se registraron 150 casos en toda la república.

De los casos notificados, la población más afectada es el grupo de 45 a 49 años. Al comparar por sexo, en 2007 se presentaba una razón de 2.9 hombres por cada mujer y para 2017 la razón disminuyó: 2.1 casos en hombres por cada mujer. 


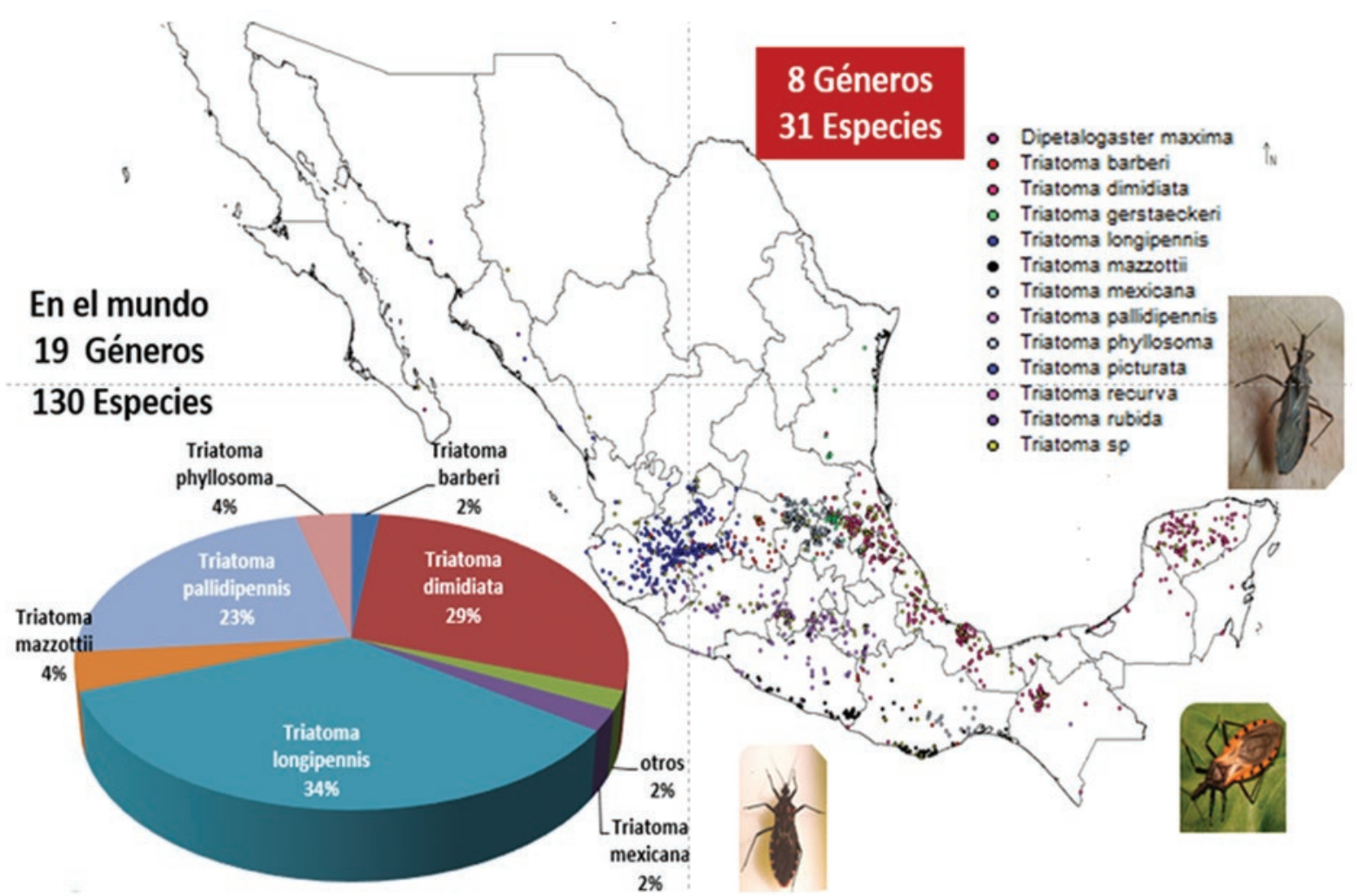

Figura 1. Distribución de triatomos en México, 2015. Fuente: Sistema Único de Información para la Vigilancia Epidemiológica, Secretaría de Salud.

Para 2017, las mayores tasas de incidencia del país se registraron en Yucatán (4.0), Oaxaca (2.4) e Hidalgo (2.1). La tasa de incidencia nacional fue de 0.7 por cada 100000 habitantes. El estado de Guerrero presentó la tasa más alta de casos nuevos en fase aguda. En su fase crónica se registraron casos en casi todos los estados, con excepción de las Baja Californias, Guerrero y Querétaro. En Yucatán se observó la tasa más alta (3.96).

En 2018, las entidades con mayor tasa de incidencia son Yucatán (0.8), Quintana Roo (0.8) y Nayarit (0.7).

La tasa de mortalidad en 2016 fue de 0.02 para hombres y 0.01 para mujeres.

Para el diagnóstico de esta infección se desarrollaron técnicas serológicas utilizando preparaciones antigénicas propias y técnicas de inmunofluorescencia indirecta, establecidas como metodologías de referencia.

Para el diagnóstico por laboratorio, en la fase aguda los estudios se centran en la búsqueda y reconocimiento del $T$. cruzi en sangre (abordaje parasitológico), ya que en las etapas iniciales de la enfermedad se encuentran parasitemias importantes. En la fase crónica (asintomática o sintomática) las parasitemias son transitorias y por ello el diagnóstico se realiza fundamentalmente mediante la búsqueda de anticuerpos circulantes contra el T. cruzi (abordaje serológico) ${ }^{6}$ (Tabla 1).
La experiencia en Sudamérica y México señala que el mejor par serológico es un ELISA confeccionado con antígenos crudos más otro ELISA con antígenos recombinantes. Las técnicas de biología molecular han mostrado variabilidad importante y a la fecha solo son de utilidad en casos particulares.

México cuenta con la Red Nacional de Laboratorios de Salud Pública, con capacidad de realizar el diagnóstico en todas las entidades de la República y que generalmente utiliza los antígenos crudos y el parásito íntegro como fuente de antígenos recombinantes.

De 2001 a 2017 se han procesado 33277 muestras en el Instituto de Diagnóstico y Referencia Epidemiológicos de la Dirección General de Epidemiología, Secretaría de Salud; 48 \% (15 mil 878) mostró positividad a enfermedad de Chagas.

Desde la década de 1990 se han logrado adelantos importantes en el control del parásito y del vector en América Latina, específicamente en México. Además, el riesgo de transmisión por transfusión sanguínea ha disminuido debido al tamizaje en todos los bancos de sangre de los países de Latinoamérica. ${ }^{7}$

La contribución en canalizar a los pacientes a las áreas de jurisdicción por parte del Centro Nacional de la Transfusión Sanguínea y de los centros estatales ha favorecido el registro de casos probables y evitado la transmisión por transfusión sanguínea. 
Gaceta Médica de México. 2018;154

Tabla 1. Obtención y manejo de muestras clínicas para el diagnóstico de enfermedad de Chagas

\begin{tabular}{|c|c|c|c|c|}
\hline Tipo de muestra & Método & Medio/contenedor/forma de envío & Tiempo & Técnica \\
\hline \multicolumn{5}{|c|}{ A.Técnicas parasitoscópicas } \\
\hline Sangre capilar & Por punción digital & $\begin{array}{l}\text { Extendido sobre portaobjetos si es posibles } \\
\text { coloreado con Giemsa, muestra de sangre en } \\
\text { laminilla a TA }\end{array}$ & $\begin{array}{l}\text { Durante la fase aguda } \\
\text { de la enfermedad }\end{array}$ & Frotis, gota gruesa \\
\hline Sangre total & $\begin{array}{l}\text { Por venopunción en tubos } \\
\text { con heparina/EDTA }\end{array}$ & $2 \mathrm{~mL}$, enviar con refrigerantes de 4 a $8^{\circ} \mathrm{C}$ & $\begin{array}{l}\text { Durante la fase aguda } \\
\text { de la enfermedad }\end{array}$ & Frotis, gota gruesa \\
\hline Sangre total & $\begin{array}{l}\text { Por venopunción en tubos } \\
\text { con heparina/EDTA }\end{array}$ & $2 \mathrm{~mL}$, enviar con refrigerantes de 4 a $8^{\circ} \mathrm{C}$ & $\begin{array}{l}\text { Durante la fase aguda } \\
\text { de la enfermedad }\end{array}$ & $\begin{array}{l}\text { Microhematócrito } \\
\text { flourescente }\end{array}$ \\
\hline Sangre total & $\begin{array}{l}\text { Por venopunción en tubos } \\
\text { con heparina }\end{array}$ & 3 a $5 \mathrm{~mL}$, enviar con refrigerantes de 4 a $8^{\circ} \mathrm{C}$ & $\begin{array}{l}\text { Durante la fase aguda } \\
\text { de la enfermedad }\end{array}$ & Hemocultivo \\
\hline Sangre total & $\begin{array}{l}\text { Por venopunción en tubos } \\
\text { con heparina }\end{array}$ & $2 \mathrm{~mL}$, enviar con refrigerantes de 4 a $8^{\circ} \mathrm{C}$ & $\begin{array}{l}\text { Durante la fase aguda } \\
\text { de la enfermedad }\end{array}$ & $\begin{array}{l}\text { Inoculación en } \\
\text { ratón }\end{array}$ \\
\hline \multicolumn{5}{|c|}{ B.Técnicas inmunoserológicas } \\
\hline Suero & $\begin{array}{l}\text { Por venopunción en tubos } \\
\text { con anticoagulantes }\end{array}$ & $1 \mathrm{~mL}$, enviar con refrigerantes de 4 a $8^{\circ} \mathrm{C}$ & $\begin{array}{l}\text { Durante la fase aguda } \\
\text { tardía y crónica de la } \\
\text { enfermedad }\end{array}$ & \\
\hline
\end{tabular}

Fuente: Lineamientos para la vigilancia epidemiológica de Chagas por laboratorio. Referencia 7.

\section{Seroprevalencia de $T$. Cruzi en bancos de sangre}

México es uno de los países considerados como endémicos por la Organización Panamericana de la Salud para la enfermedad de Chagas. Existen dos tipos de mecanismos de transmisión: la connatal y la urbana, de esta última se deriva la transmisión transfusional.

En México, en 2017 se habían registrado 596 bancos de sangre y con base en los factores de riesgo de seropositividad se ha desarrollado un cuestionario ad hoc para la entrevista a los donantes de sangre, además de mostrar el triatomino para su reconocimiento.,9

Actualmente, derivado de las modificaciones a la Norma Oficial Mexicana NOM-253-SSA-2012, para la disposición de sangre humana y sus componentes con fines terapéuticos, el tamizaje para la detección de la enfermedad de Chagas en bancos de sangre en México es de observancia obligatoria, con una cobertura actual de $100 \%,{ }^{8}$ cuando antes solo se realizaba en zonas endémicas.

Aunado a estas acciones, la confiabilidad de estos datos fue reforzada por programas como el de Control de Calidad Externo organizado por el Centro Nacional de la Transfusión Sanguínea, con $88.2 \%$ de participación.

Un estudio de 11 años monitoreó la seroprevalencia de $T$. Cruzi en los bancos de sangre mexicanos, que se midió mediante la detección de anticuerpos contra $T$. cruzi en donantes. De acuerdo con los reportes mensuales enviados al Centro Nacional de la Transfusión Sanguínea, para T. cruzi entre 2007 y 2017 fueron estudiadas 18256144 unidades de sangre donada en 516 bancos de sangre del Sistema de Salud en México, de las cuales resultaron repetidamente reactivas 70322 pruebas de serología estudiadas (Tabla 2).

Durante el periodo de estudio, de los 516 bancos de sangre, 238 fueron bancos de sangre privados (46\%), seguidos por 100 de la Secretaría de Salud (19\%), 68 del Instituto Mexicano del Seguro Social (13\%), 48 del Instituto de Seguridad y Servicios Sociales de los Trabajadores del Estado (9\%), 27 de los servicios estatales (5\%), 11 de Petróleos Mexicanos (3\%), ocho de hospitales universitarios $(2 \%)$, seis de la Cruz Roja (1.1\%), tres de la Secretaría de la Defensa Nacional $(0.6 \%)$, cuatro del Sistema Nacional para el Desarrollo Integral de la Familia $(0.8 \%)$ y tres de la Secretaría de Marina $(0.6 \%)$.

Se observó disminución de la seroprevalencia de T. cruzi de 0.37 en 2017, respecto a 0.40 en 2007 en donadores de sangre.

En el último panel del Programa de Control de Calidad Externo enviado en 2017 se reportaron 14 resultados falsos negativos; a los bancos de sangre con estos resultados se les otorgó asesoría técnica.

Debido a lo expuesto se considera que los bancos de sangre constituyen un área de oportunidad para la detección temprana y de referencia de pacientes a la jurisdicción sanitaria para diagnóstico, tratamiento y seguimiento de la enfermedad de Chagas. ${ }^{9}$ 
Tabla 2. Seroprevalencia de Tripanosoma cruzi en donadores de sangre en México, en un periodo de 11 años

\begin{tabular}{|c|c|c|c|c|}
\hline $\begin{array}{l}\text { Año de } \\
\text { estudio }\end{array}$ & $\begin{array}{l}\text { Unidades de sangre } \\
\text { total estudiadas }\end{array}$ & $\begin{array}{l}\text { Porcentaje de sangre } \\
\text { tamizada para } T \text {. cruzi }\end{array}$ & $\begin{array}{l}\text { Número de casos } \\
\text { repetidamente reactivos }\end{array}$ & Prevalencia \\
\hline 2007 & 821288 & 54.1 & 3303 & 0.40 \\
\hline 2008 & 1041487 & 64.4 & 4564 & 0.44 \\
\hline 2009 & 1367742 & 82.2 & 5498 & 0.40 \\
\hline 2010 & 1469163 & 86.5 & 5550 & 0.45 \\
\hline 2011 & 1568166 & 88.8 & 6407 & 0.41 \\
\hline 2012 & 1604027 & 92.6 & 7223 & 0.45 \\
\hline 2013 & 1679409 & 97 & 6585 & 0.39 \\
\hline 2014 & 1811090 & 92.8 & 6963 & 0.38 \\
\hline 2015 & 2135081 & 99.3 & 7961 & 0.37 \\
\hline 2016 & 2356387 & 100 & 7482 & 0.32 \\
\hline 2017 & 2402304 & 100 & 8796 & 0.37 \\
\hline Total & 18256144 & - & 70322 & 0.39 Media \\
\hline
\end{tabular}

Concentrado anual de unidades de sangre total estudiada, porcentaje de sangre total tamizada para T. cruzi, número de casos repetidamente reactivos y prevalencia anual de reactividad contra T. cruzi 2007-2017. Fuente: Informe mensual de los bancos de sangre del Sistema de Salud en México. Centro Nacional de la Transfusión Sanguínea. (Prevalencia por cada 100).

La disminución en la seroprevalencia de la enfermedad de Chagas se ha logrado realizando mejoras en el proceso de selección al donante con un cuestionario ad hoc y mostrando el triatomino al donante. ${ }^{10}$

La información obtenida ha derivado en propuestas de políticas de salud. El Centro Nacional de la Transfusión Sanguínea está colaborando en la elaboración del Manual de diagnóstico y tratamiento de la enfermedad de Chagas, cuyas aportaciones son establecer el flujograma de atención al donador (Figura 2), realizar el tamizaje a los donantes excluidos y solicitar a las autoridades locales competentes que ratifiquen los casos doblemente reactivos, a fin de acortar el tiempo de entrega de resultados.

Finalmente, dada la diversidad de especies de triatómino en México, es necesario contar con una prueba de tamizaje para bancos de sangre con antígenos elaborados en el país, para evitar resultados falsos negativos. ${ }^{11,12}$

Compartir la información permite la elaboración de programas multisectoriales con objetivos comunes en beneficio de la población mexicana.

\section{Clínica y tratamiento de la enfermedad de Chagas}

Para comprender los cuadros clínicos de la enfermedad de Chagas es fundamental considerar la gran capacidad de T. cruzi para invadir cualquier célula del organismo, con excepción del eritrocito. Las células "blanco" del parásito las constituyen las neuronas, las células miocárdicas y de músculo liso. En las dos primeras actúa simultáneamente. En el electrocardiograma se valúan los datos de destrucción neuronal, en tanto que el ecocardiograma identifica la destrucción muscular. La asociación de la destrucción de músculo liso y neuronal define la presentación de los síndromes megachagásicos (esófago, colón, raramente duodeno, estómago, yeyuno, vesícula, vejiga y otros).

Las manifestaciones iniciales de la enfermedad pasan desapercibidas en la mayoría de los casos agudos, $5 \%$ presenta signos y síntomas aparentes y $30 \%$ de estos, sin síntomas o signos visibles evolucionarán a la fase crónica asintomática; de dichos casos asintomáticos, $30 \%$ desarrollará cuadro clínico crónico con miocardiopatía o problemas digestivos.

El cuadro agudo, cuya duración es de dos a ocho semanas, se caracteriza por parasitemia; también se identifica fiebre sin características especiales. Otros signos y síntomas clínicos frecuentes son linfoadenopatías, edema subcutáneo, hepatomegalia, esplenomegalia y trastornos del ritmo cardiaco. En $5 \%$ de los pacientes se evidencian signos que permiten identificar el sitio de inoculación: el signo de Romaña, edema bipalpebral, unilateral, no doloroso; el chagoma de inoculación, lesión indurada, indolora, con cambios de coloración aparentes como eritema o un tono violáceo, y que puede aparecer en cualquier parte del cuerpo. Los ganglios homolaterales, 


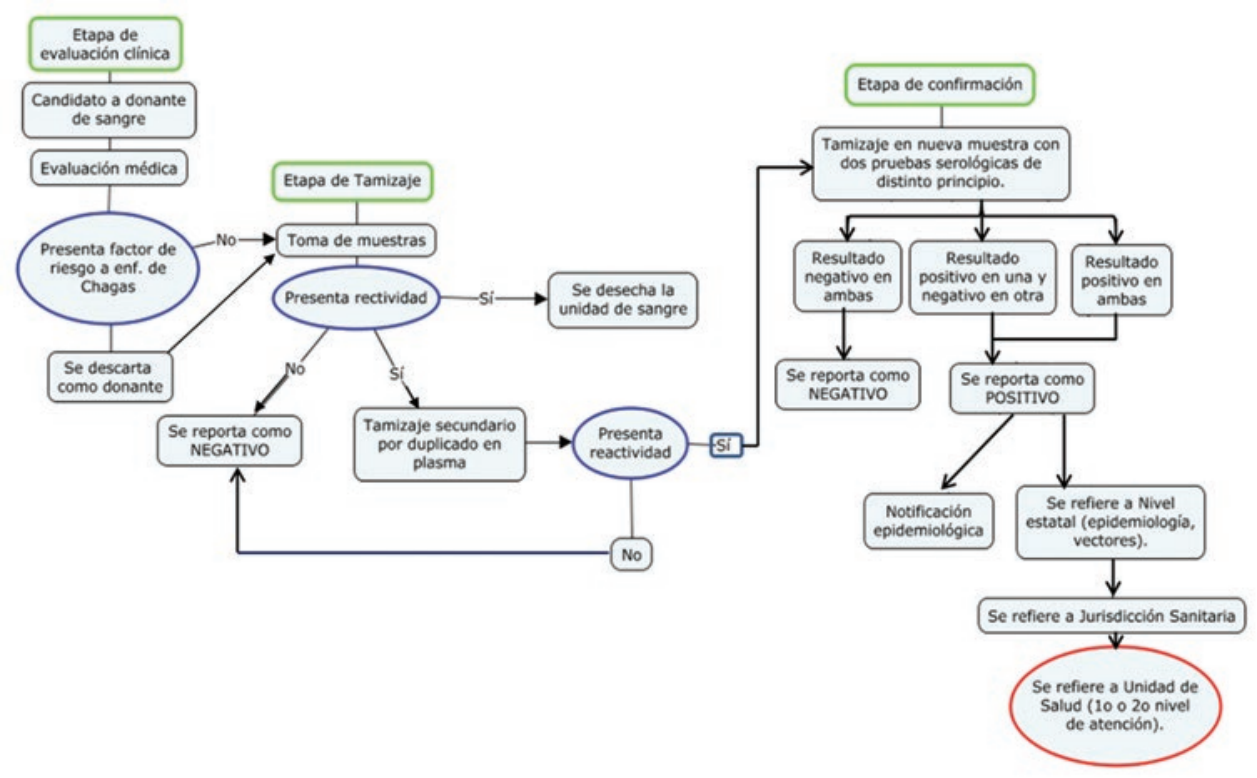

Figura 2. Flujograma de atención al donante de sangre. Manual de Diagnóstico y Tratamiento de la Enfermedad de Chagas. Subsecretaría de Prevención y Promoción a la Salud, Centro Nacional de Programas Preventivos y Control de Enfermedades.

preauricular y submaxilar, aumentan en tamaño. Suele manifestarse prurito intenso. El complejo se presenta con una duración mayor a la semana.

Algunos autores indican que la fase indeterminada 0 cuadro crónico asintomático puede tener una duración mayor a 30 años, si bien en general se considera una persistencia de 15 a 20 años. Los pacientes en esta fase no presentan síntomas o signos compatibles con la enfermedad y son detectados únicamente mediante estudios epidemiológicos y serológicos; estos pacientes constituyen un riesgo importante en la transmisión, principalmente en la vectorial y transfusional, ya que el parásito persiste en el individuo.

El cuadro clínico principal depende del compromiso cardiaco. La miocardiopatía significa un compromiso visceral irreversible. Esta patología se manifiesta en la mayor parte de los pacientes como un cuadro de miocardiopatía dilatada, pero en ocasiones emerge como una insuficiencia cardiaca congestiva, en la cual el paciente presenta disnea progresiva, palpitaciones, taquicardia, algias; en ocasiones puede haber una sola manifestación, como la taquicardia.

El trazo electrocardiográfico más frecuente en adultos de la tercera o cuarta década de la vida es el bloqueo incompleto de la rama derecha del haz de His, con hemibloqueo anterior izquierdo y extrasístoles ventriculares, en tanto que en personas más jóvenes lo usual es el bloqueo aurículo ventricular incompleto y que puede evolucionar a bloqueo completo.
El tratamiento de estos pacientes con miocardiopatía debe seleccionarse con cautela porque pueden dar lugar a mayores complicaciones.

Entre las manifestaciones megadigestivas es más frecuente el megaesófago, ${ }^{13}$ que puede diagnosticarse mediante imagen radiológica; de esta forma se identificó el primer caso en México, un paciente de 24 años del estado de Oaxaca, con el vector Triatoma barberi. El espasmo del cardias produce la dilatación del esófago; los síntomas consisten principalmente en odinofagia o disfagia, que inicia con alimentos sólidos y posteriormente con líquidos, halitosis y otros síntomas. La dilatación puede ser tan severa que se pierde el tejido interno.

El primer caso de megacolon descrito en México, en Oaxaca, se diagnosticó por un cuadro de constipación de tres semanas, con la formación de un fecaloma; el único tratamiento es la cirugía..$^{14} \mathrm{Al}$ igual que en esófago, se produce espasmo en el área afectada.

Las complicaciones en la fase aguda se presentan en menos de $1 \%$, principalmente en niños y ancianos, y consisten en miocardiopatía aguda de alta mortalidad y meningoencefalitis. En la fase crónica se puede presentar muerte súbita, registrada en 55 a $65 \%$. Otras complicaciones son la insuficiencia cardiaca progresiva, tromboembolia y aneurisma en la punta del corazón, signo patognomónico de esta enfermedad observado en México.

Los dos tratamientos que existen en la actualidad solo tienen efecto en la fase aguda, ya que actúan sobre el parásito en sangre; en la fase crónica, debido 
a la destrucción miocárdica, el tratamiento farmacológico no tiene efecto. El benznidazol es un compuesto nitroheterocíclico que actúa sobre la síntesis de ADN de $T$. cruzi, principalmente sobre tripomastigotes; se ha utilizado en la infección aguda y materno-fetal. El nifurtimoxun nitrofurano oral inhibe la síntesis de ácidos nucleicos de los tripomastigotes, principalmente mediante la formación de radicales libres de oxígeno y se utiliza en casos agudos y crónicos en los que aparentemente se disminuyen las extrasístoles. La experiencia con ambos fármacos es limitada.

La miocardiopatia chagásica infantil, descrita en la actualidad únicamente en México, se ha reportado en las huastecas veracruzana y potosina, donde se ha identificado Triatoma dimidiata, y en Querétaro, donde el triatomino identificado es Triatoma barberi; estos son los dos únicos transmisores intradomiciliriarios por excelencia, los más efectivos en la transmisión que existen en el país; Triatoma barberi es un vector que solo existe en México. ${ }^{15}$

\section{Prevención y vigilancia}

Hasta 2012, la enfermedad de Chagas se incluía entre el grupo genérico de "otras enfermedades transmitidas por vector" y las acciones para su control se limitaban a la detección y tratamiento de casos, sin embargo, reconociendo la magnitud y trascendencia de este padecimiento, a partir de 2013 se dispone del Programa de Acción Específico para la Vigilancia Prevención y Control de la Enfermedad de Chagas (PAE Chagas 2013-2018) para iniciar actividades encaminadas a eliminar la transmisión transfusional y congénita y a controlar la transmisión vectorial. $^{16}$

\section{Componentes del PAE 2013-2018. Enfermedad de Chagas}

EI PAE de Chagas 2013-2018 expone dos objetivos fundamentales:

1. Controlar la transmisión vectorial intradomiciliaria mediante la estratificación de riesgo, el mejoramiento de la vivienda y el control químico del vector.

2. Eliminar la transmisión de Chagas connatal y transfusional, fortaleciendo la coordinación interinstitucional con las áreas de Equidad de Género y Salud Reproductiva y con el Centro Nacional de la Transfusión Sanguínea. ${ }^{16,17}$

\section{Control de la transmisión vectorial introdomiciliaria}

El programa adoptó la estrategia "ruta inversa", recomendada por la Organización Panamericana de Salud/Organización Mundial de la Salud, que consiste en aplicar pruebas diagnósticas a población en riesgo e implementar acciones para el control del vector domiciliado en las localidades con focos positivos y dar seguimiento epidemiológico a casos conocidos, a sus tratamientos, a la respuesta terapéutica, y a casos nuevos. ${ }^{18}$ En la "ruta tradicional" se partía de la búsqueda de vectores infectados para ubicar focos de una enfermedad crónica y no del caso confirmado. ${ }^{19,20}$

Actualmente el control vectorial se realiza dos veces al año en localidades con presencia de triatominos, actividad que debe reforzarse con el mejoramiento de la vivienda.

Respecto a la vigilancia entomológica, 17 estados han aplicado encuestas de presencia-ausencia, con la que se ha encontrado que en las localidades de riesgo $24.23 \%$ de las viviendas aloja triatominos.

\section{Eliminación de la transmisión connatal y transfusional}

Se realiza la vigilancia prenatal y el tamizaje de mujeres embarazadas en las localidades serológicamente positivas y se debe certificar al Centro Nacional de la Transfusión Sanguínea como laboratorio de diagnóstico de Chagas para apoyar el trabajo de los laboratorios estatales de salud pública y del Instituto de Diagnóstico y Referencia Epidemiológicos. Esta vigilancia indica que la positividad serológica fue de $2 \%$ en el año 2017, la cual no se ha incrementado desde 2014.

Respecto a la transmisión transfusional, con las acciones del Centro Nacional de la Transfusión Sanguínea en los bancos de sangre y los centros estatales, se contribuye a la eliminación mediante la regulación de la obligatoriedad del tamizaje de los donadores de sangre..$^{21,22}$

La capacitación continua en los distintos niveles de atención y el manejo de pacientes en tratamiento son prioritarios para este programa, además, por medio de las jurisdicciones sanitarias se asegura el tratamiento y seguimiento de los pacientes que han dado positivo para T. cruzi. En 2017 se logró el registro del benznidazol ante la Comisión Federal para la Protección contra Riesgos Sanitarios, por considerarlo el 
fármaco de primera elección para el tratamiento de pacientes con enfermedad de Chagas. ${ }^{23,24}$

\section{Perspectivas}

La detección oportuna y el tratamiento etiológico temprano, junto con la ampliación de la cobertura, harán posible la atención médica exitosa de los pacientes con enfermedad de Chagas.

El monitoreo del tratamiento, la identificación de posibles efectos adversos a los antichagásicos y la comprobación de los resultados terapéuticos mediante pruebas de laboratorio son aspectos de la farmacovigilancia que es necesario fortalecer. La consolidación del trabajo entomológico, el control de vector y el mejoramiento en la calidad de la vivienda permitirán controlar la transmisión intradomiciliaria de la enfermedad de Chagas, de la misma manera que la vigilancia, el tamizaje de las embarazadas que viven en zonas de riesgo y el tamizaje universal de donadores de sangre y órganos harán posible la eliminación de la transmisión connatal y transfusional.

\section{Agradecimientos}

A la Universidad Nacional Autónoma de México, por el financiamiento DGAPA-PAPIIT IN-227816.

\section{Bibliografía}

1. Guadalupe-Pérez SM, Hernández-Meléndrez E, Rodríguez-Cabrera A La enfermedad de Chagas como un rezago social en salud. Rev Cubana Salud Pública. 2011;37:59-174. Disponible en: http://scielo.sld.cu/ scielo.php?script=sci_arttext\&pid=S0864-34662011000100014\&lng=es

2. Recorrido histórico. [Internet]. Hablamos de Chagas. Disponible en: http://www.hablamosdechagas.com.ar/info-chagas/recorrido-historico

3. Uribarruen-Berrueta T. Enfermedad de Chagas. México: Universidad Nacional Autónoma de México/Facultad de Medicina/Departamento de Microbiología y Parasitología. Disponible en: http://www.facmed.unam. $\mathrm{mx} /$ deptos/microbiologia/parasitologia/trypanosomosis.html

4. World Health Organization. [Sitio web]. Chagas disease (American trypanosomiasis). World Health Organization; 2018. Disponible en: http:// www.who.int/en/news-room/fact-sheets/detail/chagas-disease-(american-trypanosomiasis)

5. Organización Panamericana de la Salud/Organización Mundial de la Salud. [Sitio web] Mapa de distribución vectorial de Chagas en América Latina 2014. Disponible en: https://www.paho.org/hq/dmdocuments/2014/ Map-int-trans-vector-chagas.pdf

6. Secretaría de Salud. Norma oficial mexicana NOM-017-SSA2-2012, para la vigilancia epidemiológica. Diario Oficial de la Federación 2013 Feb 19. Disponible en: http://dof.gob.mx/nota_detalle.php?codigo=5288225\&fecha $=19 / 02 / 2013$
7. Instituto de Diagnóstico y Referencia Epidemiológicos. Lineamientos para la vigilancia epidemiológica de Chagas por laboratorio. México: Secretaría de Salud/Dirección General de Epidemiología/Instituto de Diagnóstico y Referencia Epidemiológicos; 2015. Disponible en: https:// www.gob.mx/cms/uploads/attachment/file/159071/Lineamientos_para la_vigilancia_epidemiologica_de_la_enfermedad_de_chagas.pdf

8. Norma Oficial Mexicana NOM 253-SSA1-2012, para la disposición de sangre humana y sus componentes con fines terapéuticos. México: Diario Oficial de la Federación 2012 Oct 26. Disponible en: http://www. dof.gob.mx/nota_detalle.php?codigo=5275587\&fecha=26/10/2012

9. Manual de diagnóstico de la infección por Trypanosoma cruzi. México 2006.

10. Schmunis GA. La tripanosomiasis americana como problema de salud pública. En: La enfermedad de Chagas y el sistema nervioso. Washington, D. C.: Organización Panamericana de la Salud, Publicación científica núm. 547, 1994. p. 3-31.

11. Ramos-Ligonio A, Ramírez-Sánchez ME, González-Hernández JC, Rosales-Encina JL, López-Monteon A. Prevalencia de anticuerpos contra Trypanosoma cruzi en donadores de sangre del IMSS, Orizaba, Veracruz, México. Salud Publica Mex. 206;48(1):13-21.

12. Salazar-Schettino PM, Bucio-Torres MI, Cabrera-Bravo M, De-Alba-Alvarado MC, Castillo-Saldaña DR, Zenteno-Galindo EA, et al. Enfermedad de Chagas en México. Rev Fac Med UNAM. 2016;59:6-16.

13. Salazar-Schettino PM, Tay J, Bucio MI, De-Haro I, Anzures ME, Flores-Ayala S. Primer caso de megaesófago con serología positiva a Trypanosoma cruzi. Salud Publica Mex. 1984;26:452-455.

14. Tay J, Salazar-Schettino PM, Ontiveros A, Jiménez J, De-Haro I, García-Yáñez Y, et al. Epidemiologic study of Chagas' disease in a town in Oaxaca, Mexico. Bull Pan Am Health Organ. 1986;20:358-365.

15. Salazar-Schettino PM, Cabrera-Bravo $M$, Vázquez-Antona $C$, Zenteno $E$, Alba-Alvarado MC, Torres-Gutiérrez E, et al. Chagas disease in Mexico: report of 14 cases of chagasic cardiomyopathy in children. Tohoku J Exp Med. 2016;240:243-249.

16. Secretaría de Salud. Programa Sectorial de Salud 2013-2018. México: Secretaría de Salud; 2013.

17. Secretaría de Salud. Manual de procedimientos estandarizados para la vigilancia epidemiológica de las enfermedades transmitidas por vectores. México: Secretaría de Salud/Subsecretaría de Prevención y Promoción a la Salud/Dirección General Adjunta de Epidemiología; 2017.

18. Organización Panamericana de la Salud. Suministro de sangre para transfusiones en los países del Caribe y de Latinoamérica en 2006 y 2007. Avance desde 2005 del Plan Regional de Seguridad Transfusional. EE. UU.: Organización Panamericana de la Salud; 2009.

19. Organización Panamericana de la Salud. Definición de variables y criterios de riesgos para la caracterización epidemiológica e identificación de áreas prioritarias en el control y vigilancia de la transmisión vectorial de la enfermedad de Chagas. Colombia: Comisión Intergubernamental de la Iniciativa Andina de Control de la Transmisión Vectorial y Transfusional de la Enfermedad de Chagas/Universidad de los Andes/Organización Panamericana de la Salud; 2004.

20. Organización Panamericana de la Salud. Enfermedades infecciosas desatendidas en las Américas: historia de éxito e innovación para llegar a los más necesitados. EE. UU.: Organización Panamericana de la Salud; 2016.

21. Silveira AC. Programa regional para el control de la enfermedad de Chagas en América Latina. Comisión Nacional Honoraria de Zoonosis/ Organización Panamericana de la Salud/Banco Interamericano del Desarrollo. Disponible en https://www.paho.org/per/index.php?option=com docman\&view=download\&alias=261-programa-regional-para-control-enfermedad-chagas-america-latina-lineamientos-recomendaciones-tecnicas-politica-publica-para-abordaje-enfermedad-chagas-1\&category_slug=chagas-998\&ltemid=1031

22. Guzmán-Bracho C, García-García L, Floriani-Verdugo J, Guerrero-Martínez S, Torres-Cosme M, Ramírez-Melgar C, et al. Riesgo de transmisión de Tripanosoma cruzi por transfusión de sangre en México. Panam Salud Publica. 1998;4:94-98.

23. Organización Panamericana de la Salud. Enfermedad de Chagas en las Américas: una revisión actual de salud pública y su visión para el futuro. Ginebra, Suiza: Organización Panamericana de la Salud; 2018.

24. Organización Panamericana de la Salud. Enfermedades tropicales desatendidas. Ginebra, Suiza: Organización Mundial de la Salud; 2018. Disponible en: http://www.who.int/topics/tropical_diseases/qa/faq/es 